\title{
Progranulin and Activin A Concentrations are Elevated in Serum from Patients with Acute Exacerbations of Idiopathic Pulmonary Fibrosis
}

\author{
Tian Xie ${ }^{1}\left[\right.$ Lizhen $\mathrm{Han}^{2} \cdot$ Yongxing Chen ${ }^{1} \cdot$ Haihong $\mathrm{Wu}^{1}$
}

Received: 11 April 2021 / Accepted: 19 August 2021 / Published online: 30 August 2021

(c) The Author(s) 2021

\begin{abstract}
Purpose Idiopathic pulmonary fibrosis (IPF) is a chronic, progressive fibrotic lung disease of unknown cause with a variable course. Acute exacerbations of IPF (AE-IPF) is sudden accelerations of the disease or a superimposed idiopathic acute injury significantly reducing lung function. To examine the serum concentrations of Progranulin (PGRN) and activin A in patients with AE-IPF in a pilot study.

Methods Twenty-one patients with AE-IPF were compared with 23 patients with stable IPF as a control group. Serum PGRN and activin A levels, arterial blood gas measurements, and lung function were determined in these two groups.

Results Peripheral blood PGRN and activin A levels in patients with AE-IPF were $83.7+10.0$ and $14.2 \pm 1.7 \mathrm{ng} / \mathrm{ml}$ $($ mean $+\mathrm{SD})$, respectively; higher than those in the control group $61.0+5.8$ and $5.8+1.0(p<0.001)$. PGRN and activin A levels were significantly negatively correlated with carbon monoxide diffusion capacity $r=-0.857(p<0.001)$ and $r=-0.757$ $(p<0.001)$.
\end{abstract}

Conclusion Progranulin (PGRN) and activin A may be involved in the pathogenesis of AE-IPF. They may be possible markers of disease activity in AE-IPF.

Keywords Idiopathic pulmonary fibrosis · Acute exacerbation · Progranulin · Activin A

\section{Introduction}

Idiopathic pulmonary fibrosis (IPF) is a chronic, progressive, and fibrotic interstitial pneumonia, the pathogenesis of which is not fully understood. There are currently no very effective therapeutic interventions other than lung transplantation, although pirfenidone and nintedanib are thought to slow deterioration of lung function and may reduce the frequency of acute exacerbations to some extent. The overall

Lizhen Han is co-first author and contributed equally in this work.

Tian Xie

girlxietian@sina.com

1 Department of Pulmonary and Critical Care Medicine, Hainan General Hospital, Hainan Affiliated Hospital of Hainan Medical University, 19 Xiuhua Road, Xiuying District, Haikou 570311, Hainan Province, China

2 Department of Medical Records and Statistics, Hainan General Hospital, Hainan Affiliated Hospital of Hainan Medical University, Haikou 570311, Hainan, China prognosis remains poor [1]. Most patients experience progressive decline over time but the clinical course can be highly variable [2]. Acute exacerbations of IPF represents sudden deteriorations in lung function occurring due to sudden accelerations in lung disease or superimposed acute lung injury which may be idiopathic.

Progranulin (PGRN) is a secreted 539-amino acid multifunctional growth factor thought to be involved in a variety of physiological and disease processes including embryogenesis, wound healing, inflammation, tumorigenesis, and host defense. PGRN is widely expressed in immune cells such as epithelial cells, macrophages, dendritic cells, and also within neurones. It plays a pro-inflammatory role in post-injury repair, in diabetes in the presence of insulin resistance, and in obesity and an anti-inflammatory role in lipopolysaccharide-induced acute pneumonia, in acute cerebral ischemia, and in various autoimmune diseases in mice [3]. There is evidence of its involvement in wound healing and diverse conditions such as psoriasis and colitis in humans and in mouse models [4]. In a recent study serum PGRN was significantly higher in dermatomyositis 
(DM) patients than in polymyositis or healthy controls. Concentrations were significantly raised in DM combined with interstitial lung disease and may be associated with reduced 6-month survival in this group [5]. Activin A is a multipotent cytokine, a member of the transforming growth factor beta (TGF- $\beta$ ) superfamily, which is involved in inflammatory, tumor, and fibrotic processes in a variety of tissues and organs and its expression is elevated in idiopathic pulmonary fibrosis [6].

We have found no studies on PGRN or activin A in acute exacerbations of idiopathic pulmonary fibrosis, and this study aimed to examine the concentrations of PGRN and activin $\mathrm{A}$ in the serum of patients with acute exacerbations and compare them with those in stable idiopathic pulmonary fibrosis to investigate any potential pathogenetic significance. The possibility that one or both proteins might have potential as clinical markers of AE-IPF was also considered.

\section{Materials and Methods}

\section{General Data}

Patients were selected from those who were hospitalized in Hainan General Hospital for AE-IPF between January 2017 and June 2020. All met the diagnostic criteria for AE-IPF [1]: (1) Previous or concurrent diagnosis of IPF, (2) Acute worsening or development of dyspnea typically of < 1-month duration, (3) High-resolution computed tomography (HRCT) with new, bilateral, ground-glass opacification, and/or consolidation superimposed on a background pattern consistent with usual interstitial pneumonia (UIP) pattern, and (4) Deterioration not fully explained by clinically significant cardiac failure or fluid overload or pulmonary embolism. The control group included patients with stable IPF who regularly attended follow-up in the Respiratory Medicine Outpatient Clinic of Hainan General Hospital. We applied the following criteria of IPF [7]: (1) Compatible clinical features, (2) The presence of a UIP pattern on HRCT (performed according to the recommended technical requirements): subpleural, basal predominance, reticular abnormality, honeycombing with or without traction bronchiectasis, and absence of features listed as inconsistent with UIP, (3) Exclusion of other known causes of interstitial lung disease including negative connective tissue serology, and (4) Agreement of the diagnosis by all members in a multidisciplinary discussion among respiratory physicians, imaging physicians, and pathologists with rich experience in the diagnosis of ILD (which was accepted by all of the patients). None of the patients underwent surgical lung biopsy. Exclusion criteria included IPF patients with concomitant: (1) chronic obstructive pulmonary disease (COPD), asthma, bronchiectasis, pulmonary infection, pulmonary embolism, or other respiratory diseases, (2) malignancy, (3) stroke, Alzheimer's disease, acute coronary syndrome, type 2 diabetes mellitus, and obesity (BMI $\geq 30 \mathrm{~kg} / \mathrm{m}^{2}$ ), (4) renal or hepatic dysfunction, cirrhosis, and fibrosis, (5) autoimmune diseases such as psoriasis, dermatomyositis, or rheumatoid arthritis, (6) patients receiving, or who had received, immunosuppressant or glucocorticoid therapy within the previous 3 months, and (7) patients receiving, or who had received, bronchodilators within the previous 3 months.

The experimental protocol was approved by the Human Ethics Committee of Hainan General Hospital. Written informed consent was obtained from individual participants.

Twenty-one patients were included in the AE-IPF study group and 23 patients with stable IPF were enrolled as a control group.

\section{Methods}

Five milliliters of peripheral venous blood was drawn from fasting patients in both groups within $24 \mathrm{~h}$ after enrollment. Supernatants were extracted by centrifugation at $3000 \mathrm{rpm}$ for $10 \mathrm{~min}$. Serum levels of PGRN and activin A were measured using enzyme-linked immunoassay (ELISA) kits (R\&D Systems, USA) according to the product's specification. According to the kit specification, we diluted the samples before testing. Arterial blood gas analysis (Blood gas analyzer Radiometer ABL80) was performed immediately after $2 \mathrm{ml}$ sample of peripheral arterial blood was collected. Pulmonary function tests (Medisoft Micro 5000, Belgium) were performed within $48 \mathrm{~h}$ after enrollment. The main variables recorded were the measured/expected ratio of Forced Vital Capacity (FVC) and diffusion capacity for carbon monoxide (DLCO) both expressed as percent predicted. Spirometry was conducted by the same physician according to the international guidelines, using a PFT spirometer (Medisoft Micro 5000, Belgium).

\section{Statistical Analysis}

SPSS17.0 statistical software was used to analyze the patients' data. The Kolmogorov-Smirnov test was performed on continuous data. Patients' age, PGRN and activin A concentrations, blood gas measurements, FVC, and DLCO were presented as mean \pm standard deviation $(\mathrm{SD})$. The differences between groups were analyzed by Student's t test. Discrete and categorical data such as patient gender ratio and use of anti-fibrotic treatment were analyzed by $\chi 2$ test. Correlations between PGRN and activin A levels and $\mathrm{DL}_{\mathrm{CO}}$ in all patients were analyzed by Spearman correlation analysis. All tests were two-tailed. $p<0.05$ was taken as indicating a statistically significant difference. 


\section{Results}

The study group included 21 patients, 19 males and 2 females, aged between 52 and 71 with a mean age of $65.2 \pm 6.8$ years. The control group included 23 patients, 20 males and 3 females, aged between 49 and 68, with a mean age of $62.4 \pm 5.7$ years

All patients in the study group had mild AE-IPF but all patients in the study required supplemental oxygen therapy admission to hospital. There were no statistically significant differences in general characteristics such as age, gender, duration of IPF, and baseline anti-fibrotic treatment status between the two groups, see Table 1 for details $(p>0.05)$.

Mean FVC and $\mathrm{DL}_{\mathrm{CO}}$ measurements were significantly lower in the study group compared to patients with stable IPF ( $p<0.05$, see Table 2 for details). The PGRN and activin A concentration data were normally distributed by Kolmogorov-Smirnov test. The serum levels of PGRN and activin A in the study group were significantly higher than those in the control group $(p<0.05)$ (see Table 2, Figs. 1, 2). The level of A-a gradient of oxygen in the study group was significantly higher than that in the control group $(p<0.05)$.

A significant negative correlation was demonstrated between PGRN and $\mathrm{DL}_{\mathrm{CO}}$; Spearman correlation coefficient $r=-0.859, p<0.001$ indicating that serum PGRN concentration increased as $\mathrm{DL}_{\mathrm{CO}}$ decreased (see Fig. 3). A significant negative correlation was also demonstrated between activin $\mathrm{A}$ and $\mathrm{DL}_{\mathrm{CO}}$; Spearman correlation coefficient $r=-0.757, p<0.001$ indicating that serum activin A concentration increased as $\mathrm{DL}_{\mathrm{CO}}$ decreased (see Fig. 4).

\section{Discussion}

Idiopathic pulmonary fibrosis is the commonest form of idiopathic interstitial pneumonia, the pathogenesis of which is not understood, although epithelial cell injury is thought to underlie abnormal healing and subsequent fibrosis. The natural history is variable; a steady predictable decline is common but some patients experience acute deteriorations of unknown etiology termed AE-IPF. This small, singlecenter study has shown significant elevations of PGRN and activin A in patients with mild AE-IPF compared with stable IPF. No a priori power calculation was possible to determine sample size as no previous estimates of PGRN or activin A were available in this population but this was intended as a pilot study. The PGRN concentrations determined in this study were similar to those measured by Tanaka et al. in DM with ILD [5]. The negative correlations of activin A and PGRN with $\mathrm{DL}_{\mathrm{CO}}$ are of interest, although preliminary, as they could represent only linear interpolation of data from two distinct patient populations. However, if real, they may imply some cause and effect relationship; that is, whatever processes reflected by increase in these growth factors, bearing in mind their multiple possible cellular origins and their multiplicity of effects, may relate directly to acute deterioration in the diffuse alveolitis and disorganization of alveolar structures, ultimately leading to an acute exacerbation of the interstitial fibrosis. The negative correlation of PGRN and activin A with gas transfer but not with FVC is unexplained but, if genuine, could be because the changes in AE-ILD are mild reflecting inflammation or altered ventilation perfusion
Table 1 General characteristics of the enrolled patients

\begin{tabular}{lllll}
\hline & AE-IPF & Control group & $\chi^{2} / t$ & $p$-value \\
\hline Number of patients & 21 & 23 & & \\
Sex (male) & $19(90.5 \%)$ & $20(87.0 \%)$ & $\chi^{2}=0.012$ & 0.914 \\
Age (years), mean \pm SD & $65.2 \pm 6.8$ & $62.4 \pm 5.7$ & $t=1.485$ & 0.145 \\
Duration of IPF (years), mean \pm SD & $1.2 \pm 0.3$ & $1.0 \pm 0.5$ & $t=1.589$ & 0.119 \\
Baseline anti-fibrotic therapy $^{\mathrm{a}}$ & $9(42.86 \%)$ & $12(52.17 \%)$ & $\chi^{2}=0.100$ & 0.752 \\
\hline
\end{tabular}

${ }^{a}$ Baseline anti-fibrotic therapy included pirfenidone and nintedanib

\begin{tabular}{llccr}
\hline & AE-IPF & Control group & $t$ & $p$-value \\
\hline PGRN (ng/ml), mean \pm SD & $83.7 \pm 10.0$ & $61.0 \pm 7.3$ & 8.64 & $<0.001$ \\
Activin A (ng/ml), mean \pm SD & $14.2 \pm 1.7$ & $5.8 \pm 1.0$ & 19.7 & $<0.001$ \\
A-a gradient (mmHg), mean \pm SD & $38.2 \pm 5.28$ & $34.7 \pm 3.61$ & 2.58 & 0.013 \\
DLco\% pred, mean \pm SD & $33.8 \pm 5.5$ & $50.6 \pm 5.4$ & -6.79 & $<0.001$ \\
FVC\% pred, mean \pm SD & $39.4 \pm 5.8$ & $48 \pm 6.0$ & -4.566 & $<0.001$ \\
\hline
\end{tabular}

A-a gradient: the difference in blood oxygen partial pressure between alveoli and arterial capillaries DLco: carbon monoxide diffusion capacity $\%$ pred: percentage predicted

FVC: forced vital capacity $\%$ pred: percentage predicted
Table 2 Comparison of serum levels of PGRN, activin A, A-a gradient, $\mathrm{DL}_{\mathrm{CO}} \%$ pred, and $\mathrm{FVC} \%$ pred in the two groups 
Fig. 1 Plasma PGRN levels in patients with stable IPF and AE-IPF

Fig. 2 Plasma activin A levels in patients with stable IPF and AE-IPF
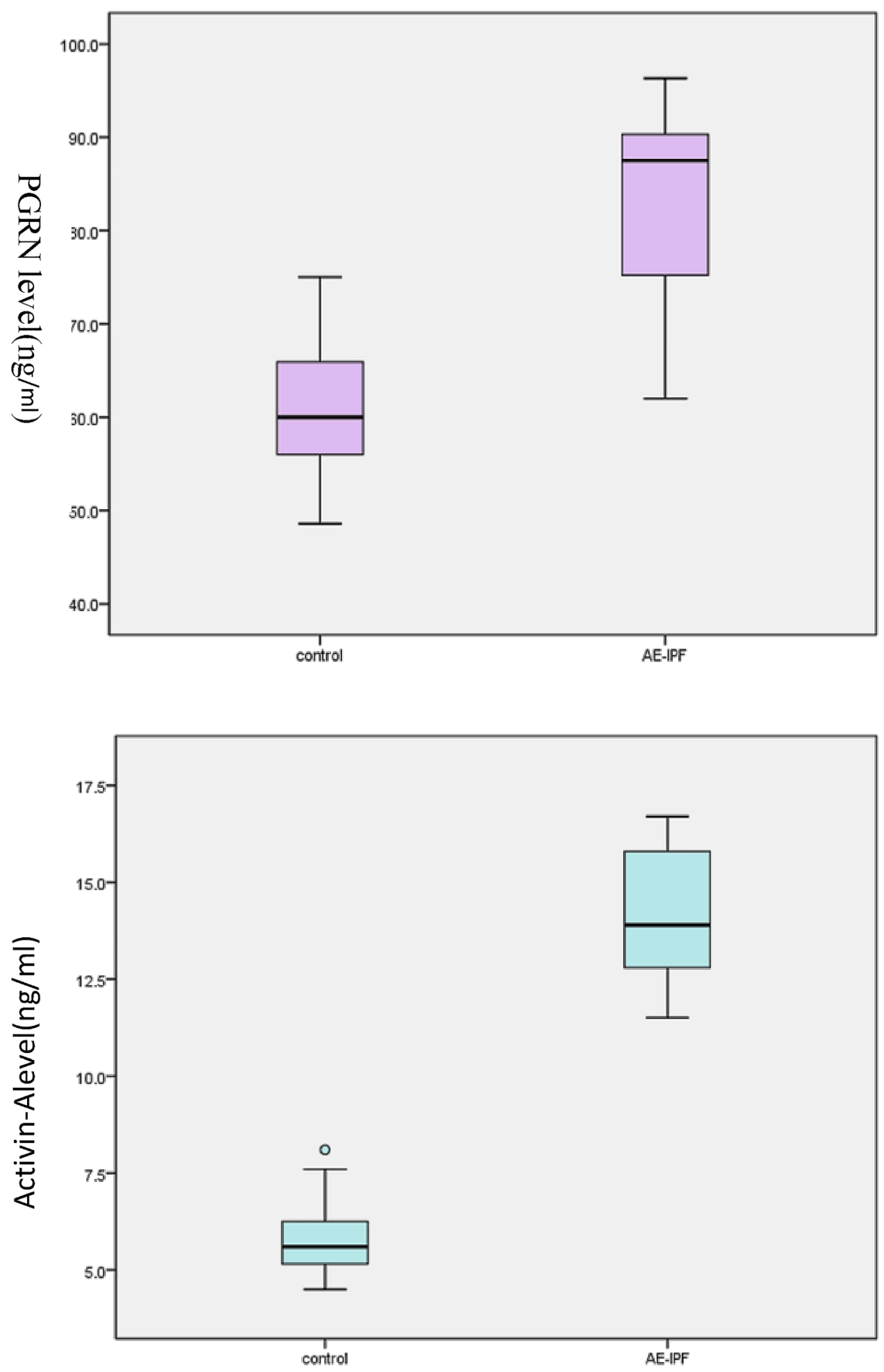

rather than factors affecting volume loss, e.g., fibrosis. The mechanism may be that PGRN and activin A are involved in the inflammatory response to common triggers of acute exacerbations of idiopathic pulmonary fibrosis such as bacterial or viral infections, gastric contents aspiration, and air pollution. Even if not mechanistically important PGRN and activin A could potentially be useful clinically as markers of AE-IPF.

Sputum PGRN concentrations have been reported to be higher in acute exacerbations of COPD (AECOPD) than in stable COPD patients or controls and to be negatively correlated with $\mathrm{FEV}_{1}$. It has been suggested that PGRN might 
Fig. 3 Correlation between PGRN levels and DLco; significant negative correlation between PGRN and DLco in IPF patients
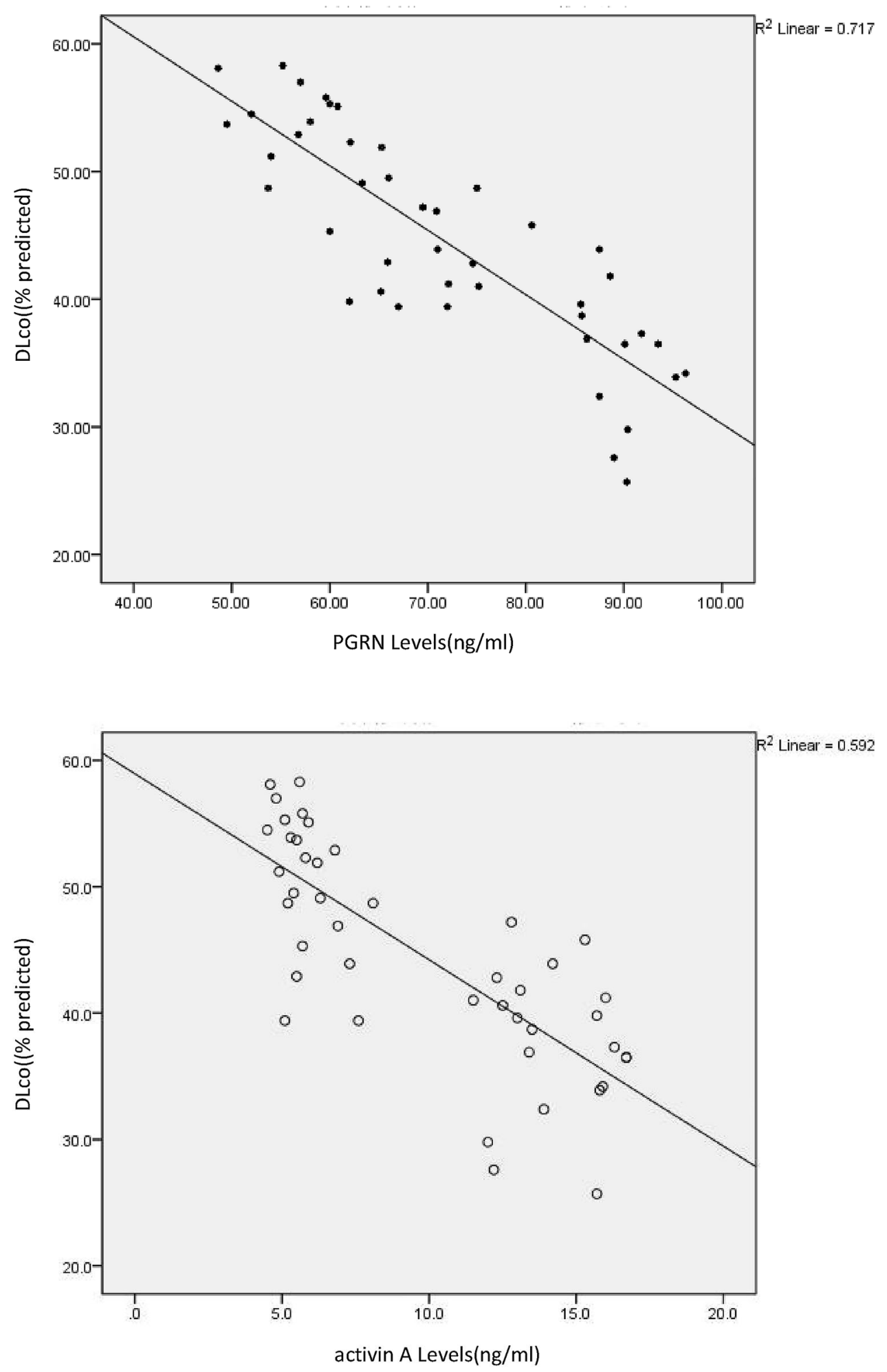

Fig. 4 Correlation between activin A levels and DLco; significant negative correlation between activin A and DLco in IPF patients be an independent predictor of AECOPD [8]. However, in asthma, including occupational asthma, serum levels were reduced compared to controls $[9,10]$. Serum PGRN concentrations were reportedly raised in acute community acquired pneumonia and correlated with poor prognosis [11]. In mouse models of cigarette smoke-induced epithelial injury [12], endotoxin shock [13], and an LPS-induced acute lung injury model [14], PGRN depletion (knockdown) increases inflammation while over-expression or pre-treatment reduces it. 
Activin A, a member of the transforming growth factor $\beta$ family, is importantly involved in angiotensin II-mediated atrial fibrosis and in promoting fibroblast differentiation of endometrial mesenchymal stem cells [15, 16]. It participates in fibrosis in liver, pancreas, and kidneys [17]. Activin A can stimulate fibroblast differentiation into myofibroblasts inducing the proliferation of lung fibroblasts, airway smooth muscle cells, and bronchial epithelial cells [18]. Levels were raised in airways severe asthma [19] and in the serum of patients with COPD compared to healthy controls [20]. Activin A and activin B are expressed at increased levels in alveolar epithelial cells and inflammatory cells in patients with idiopathic pulmonary fibrosis [6], and the activin A antagonist follicle suppressor can also reduce bleomycin-induced pulmonary fibrosis [17].

Potential weaknesses of this study are the small sample size and the limited number of relevant parameters measured. Only patients with mild IPF-AE were examined. Factors related to IPF, but also possible co-morbidities, need to be further refined to exclude bias. Measurements were made only at a single time point and there is no sequential information about evolution of the signals. Future, larger, prospective, ideally longitudinal, studies will be needed to confirm these findings and to focus on trying to dissect possible pro-inflammatory and anti-inflammatory effects of PGRN and activin A and their mechanisms in IPF and particularly in AE-IPF. Comparison of PGRN and activin A with other possible markers of disease activity in IPF, e.g., KL-6 [21], Matrix metalloproteinases 1 and 7, and Surfactant Proteins A and D, chemokines, and cytokines such as chemokine ligand 18 and interleukin 8 [22] are urgently needed to assist diagnosis and treatment of acute exacerbations of idiopathic pulmonary fibrosis.

Acknowledgements This paper was supported by the Hainan Provincial Natural Science Foundation of China (Grant No. 819QN354).

Author Contributions TX designed the research and wrote the paper. LH analyzed the data. YC collected the data. HW designed the research.

Funding This study was supported by the Hainan Provincial Natural ScienceFoundation of China (819QN354).

Data Availability All data and material generated or used during the study appear in the submitted article.

\section{Declarations}

Conflict of interest The authors confirm that there are no conflicts of interest.

Ethical Approval The experimental protocol was approved by the Human Ethics Committee of Hainan General Hospital.
Consent to Participate Written informed consent was obtained from individual participants.

Consent for Publication Written informed consent for publication was obtained from all participants.

Open Access This article is licensed under a Creative Commons Attribution 4.0 International License, which permits use, sharing, adaptation, distribution and reproduction in any medium or format, as long as you give appropriate credit to the original author(s) and the source, provide a link to the Creative Commons licence, and indicate if changes were made. The images or other third party material in this article are included in the article's Creative Commons licence, unless indicated otherwise in a credit line to the material. If material is not included in the article's Creative Commons licence and your intended use is not permitted by statutory regulation or exceeds the permitted use, you will need to obtain permission directly from the copyright holder. To view a copy of this licence, visit http://creativecommons.org/licenses/by/4.0/.

\section{References}

1. Collard HR, Ryerson CJ, Corte TJ, Jenkins G, Kondoh Y et al (2016) Acute exacerbation of idiopathic pulmonary fibrosis. An international working group report. Am J Respir Crit Care Med 194:265-275

2. Ley B, Collard HR, King TE Jr (2011) Clinical course and prediction of survival in idiopathic pulmonary fibrosis. Am J Respir Crit Care Med 183:431-440

3. Kanazawa M, Kawamura K, Takahashi T, Nishizawa M, Shimohata T (2016) Progranulin. Nihon Insho 74:579-582

4. Huang K, Chen A, Zhang X, Song Z, Xu H et al (2015) Progranulin is preferentially expressed in patients with psoriasis vulgaris and protects mice frompsoriasis-like skin inflammation. Immunology 145:279-287

5. Tanaka A, Tsukamoto H, Mitoma $\mathrm{H}$, Kiyohara $\mathrm{C}$, Ueda $\mathrm{N}$ et al (2015) Serum progranulin levels are elevated in dermatomyositis patients with acute interstitial lung disease, predicting prognosis. Arthritis Res Ther 17:27

6. Myllärniemi M, Tikkanen J, Hulmi JJ, Pasternack A, Sutinen E et al (2014) Upregulation of activin-B and follistatin in pulmonary fibrosis - a translational study using human biopsies and a specific inhibitor in mouse fibrosis models. BMC Pulm Med 14:170

7. Raghu G, Collard HR, Egan JJ, Martinez FJ, Behr J et al (2011) An official ATS/ERS/JRS/ALAT statement: idiopathic pulmonary fibrosis: evidence-based guidelines for diagnosis and management. Am J Respir Crit Care Med 183:788-824

8. Chen X, Liu J, Zhu M, Hu B, Bai F et al (2018) Progranulin is a novel biomarker for predicting an acute exacerbation of chronic obstructive pulmonary disease. Clin Respir J 12:2525-2533

9. Choi GS, Trinh HKT, Yang EM, Ye YM, Shin YS et al (2018) Role of clusterin/progranulin in toluene diisocyanate-induced occupational asthma. Exp Mol Med 50:1-10

10. Park SY, Hong GH, Park S, Shin B, Yoon SY et al (2016) Serum progranulin as an indicator of neutrophilic airway inflammation and asthma severity. Ann Allergy Asthma Immunol 117:646-650

11. Luo Q, He X, Zheng Y, Ning P, Xu Y et al (2020) Elevated progranulin as a novel biomarker to predict poor prognosis in community-acquired pneumonia. J Infect 80:167-173

12. Lee KY, Park SY, Park S, Hong GH, Moon KA et al (2017) Progranulin protects lung epithelial cells from cigarette smokinginduced apoptosis. Respirology 22:1140-1148 
13. Yu Y, Xu X, Liu L, Mao S, Feng T et al (2016) Progranulin deficiency leads to severe inflammation, lung injury and cell death in a mouse model of endotoxic shock. J Cell Mol Med 20:506-517

14. Xie W, Lu Q, Wang K, Lu J, Gu X et al (2018) miR-34b-5p inhibition attenuates lung inflammation and apoptosis in an LPSinduced acute lung injury mouse model by targeting progranulin. J Cell Physiol 233:6615-6631

15. Wang Q, Yu Y, Zhang P, Chen Y, Li C et al (2017) The crucial role of activin A/ALK4 pathway in the pathogenesis of Ang-IIinduced atrial fibrosis and vulnerability to atrial fibrillation. Basic Res Cardiol 112:47

16. Zhang Z, Wang J, Chen Y, Suo L, Chen H et al (2019) Activin a promotes myofibroblast differentiation of endometrial mesenchymal stem cells via STAT3-dependent Smad/CTGF pathway. Cell Commun Signal 17:45

17. Protic O, Islam MS, Greco S, Giannubilo SR, Lamanna P et al (2017) Activin A in inflammation, tissue repair, and fibrosis: possible role as inflammatory and fibrotic mediator of uterine fibroid development and growth. Semin Reprod Med 35:499-509

18. Hardy CL, Rolland JM, O'Hehir RE (2015) The immunoregulatory and fibrotic roles of activin A in allergic asthma. Clin Exp Allergy 45:1510-1522
19. Samitas K, Poulos N, Semitekolou M, Morianos I, Tousa S et al (2016) Activin-A is overexpressed in severe asthma and is implicated in angiogenic processes. Eur Respir J47:769-782

20. Zhou G, Gui X, Chen R, Fu X, Ji X, Ding H et al (2019) Elevated serum Activin A in chronic obstructive pulmonary disease with skeletal muscle wasting. Clinics (Sao Paulo) 74:e981

21. Ohshimo S, Ishikawa N, Horimasu Y, Hattori N, Hirohashi N et al (2014) Baseline KL-6 predicts increased risk for acute exacerbation of idiopathic pulmonary fibrosis. Respir Med 108:1031-1039

22. Guiot J, Moermans C, Henket M, Corhay JL, Louis R (2017) Blood biomarkers in idiopathic pulmonary fibrosis. Lung 195:273-280

Publisher's Note Springer Nature remains neutral with regard to jurisdictional claims in published maps and institutional affiliations. 